\title{
Centrosome Remodeling During Fertilization and Embryo Development
}

\author{
Heide Schatten $^{*}$ \\ *Dept. of Veterinary Pathobiology, University of Missouri, Columbia, MO 65211
}

Successful fertilization and embryo development significantly depends on functional centrosomes that are able to nucleate and organize microtubules for pronuclear apposition, symmetric and asymmetric cell divisions and coordinated signal transductions that allow cell cycle regulations throughout development. In most mammalian species, the sperm contributes the nucleating centrosome components containing a pair of centrioles that serves as core structure for the assembly of oocyte centrosomal proteins after sperm incorporation into the oocyte. Rapid remodeling of the zygotic centrosome is critical for sperm aster formation and subsequent microtubule organizations for the formation of the bipolar mitotic apparatus during first and subsequent cell divisions. Remodeling of centrosomes requires microtubule motor proteins and a cascade of signaling molecules for transport of centrosome proteins and kinases to restructure the zygotic centrosome into a division-competent (mature) centrosome.

Several labs including ours have identified sperm centrosome pathologies that are associated with male-factor infertility [1-3] and we have further shown that centrosome abnormalities in aging oocytes plays a role in female-factor infertility associated with aging [4-5]. In both situations, new studies have shown that some of the centrosome abnormalities can be overcome with chemical centrosome stabilization (female-factor infertility related to oocyte aging) or intracytoplasmic sperm injection (ICSI) combined with sperm-factor supplementation or under various activation conditions in model animal species. However, these studies are only at the beginning, as we do not yet fully understand the various factors that are needed for accurate centrosome functions during fertilization and subsequent development.

Our recent studies have focused on centrosome remodeling during nuclear cloning in which centrosomes from a somatic cell need to be remodeled by the activated oocyte to carry out functions that are normally fulfilled by the zygotic centrosome of sperm and eggs. Failure in centrosome remodeling may be among the underlying reasons for the low percent in cloning success which ranges from $1-5 \%$ in most animal species. We have identified $39.4 \%$ centrosome abnormalities during the first cell cycle of cloned pig embryos with multipolar mitosis rather than bipolar mitoses in control embryos [6]. Multipolar mitoses are hallmarks for cancer cells and will result in apoptosis, mitotic cell death, or abnormal cell divisions. Current studies are aimed at investigating factors involved in centrosome regulation. 


\section{References}

[1] Schatten, H., and Sun, Q-Y. Mol Human Reproduction 15(9):531-538 (2009a).

[2] Schatten, H., and Sun, Q-Y. Environ Mol Mutagen 50(8):620-638 (2009b).

[3] Schatten, H., and Sun, Q-Y. Seminars in Cell and Devel Biol, in press (2010).

[4] Miao, Y-L., Sun, Q-Y., Zhang, X., Zhao, J-G., Zhao, M-T., Spate, L., Prather, R.S., and Schatten, H. Environ Mol Mutagen 50(8):666-71 (2009).

[5] Miao, Y-L., Kikuchi, K., Sun, Q-Y., and Schatten H. Human Reprod Update 15(5):573-585 (2009).

[6] Zhong, Z., Spate, L., Hao, Y., Li, R., Lai, L., Katayama, M., Sun, Q.Y., Prather, R.S., Schatten, H. Cell Cycle 6(12):1510-1521 (2007). 